\title{
Acute motor axonal neuropathy in a case of granulomatosis with polyangiitis
}

\author{
Ioan-Cristian Lupescu' ${ }^{1}$ Mirela Ramona Draghici' ${ }^{1}$, Adriana Octaviana Dulamea ${ }^{1,2}$ \\ ${ }^{1}$ Neurology Department, Fundeni Clinical Institute, Bucharest, Romania \\ ${ }_{2}^{2}$ Carol Davila“ University of Medicine and Pharmacy, Bucharest, Romania
}

\begin{abstract}
A 65 years-old woman started developing arthralgias, mild cough and dyspnea. When symptoms progressed to productive cough and moderate exertional dyspnea, she was admitted to the hospital. Thoracic CT revealed diffuse interstitial pneumopathy, pulmonary function tests indicated moderate obstruction (FEV1 $=60 \%$ ) and bronchoalveolar lavage was suggestive of severe alveolar-hemorrhagic syndrome. Laboratory workup confirmed the presence of antibodies against myeloperoxidase (i.e. p-ANCA) and absence of mycobacterium tuberculosis, hence a diagnosis of granulomatosis with polyangiitis was established. During hospitalization, she developed rapidly progressive paraparesis with retained deep tendon reflexes. Electroneurography highlighted the presence of subacute onset motor axonal polyneuropathy affecting both upper and lower limbs. Under treatment with methylprednisolone, oral cyclophosphamide, alpha-lipoic acid and intravenous immunoglobulins, improvement of neurological status was noted.
\end{abstract}

Keywords: axonal neuropathy, granulomatosis, polyangiitis

\begin{abstract}
Abbreviations
AMAN - acute motor axonal neuropathy

ANCA - anti-neutrophil cytoplasmic autoantibody

CMAP - compound muscle action potential

CT - computer tomography

EMG - electromyography

FEV1 - forced expiratory volume in one second

GBS - Guillain Barré syndrome
\end{abstract}

\author{
MCV - motor conduction velocity \\ $\mathrm{MRC}$ - medical research council \\ MRI - magnetic resonance imaging \\ PNS - peripheral nervous system \\ SCV - sensory conduction velocity \\ SNAP - sensory nerve action potential \\ WG - Wegener granulomatosis
}

\section{INTRODUCTION}

Granulomatosis with polyangiitis (formerly known as Wegener's granulomatosis) is a type of small-vessel vasculitis included in the anti-neutrophil cytoplasmic autoantibody (ANCA)-associated vasculitides [1]. It is an autoimmune granulomatous vasculitis, in which antibodies against myeloperoxidase and proteinase 3 are revealed in the serum [2]. The clinical presentation is varied and usually includes: constitutional symptoms (e.g. fever, weight loss, anorexia, malaise); ear/ nose/throat manifestations (sinusitis, nasal septal perforation, otitis media, subglottic stenosis); pul- monary disease (pulmonary cavitary infiltrates or nodules expressed as cough, hemoptysis and dyspnea), renal disease (crescentic glomerulonephritis with rapidly progressive renal failure), cutaneous involvement (palpable purpura, papules, ulcers), cardiac involvement (pericarditis, coronary vasculitis, cardiomyopathy) and ophthalmic manifestations (conjunctivitis, scleritis, ciliary vessel vasculitis or retroorbital mass lesions) [3]. Nervous system involvement has been estimated in about $33 \%$ of patients and consists of mononeuropathy multiplex, distal symmetrical polyneuropathy, cranial neuropathy, external ophthalmoplegia, cerebrovascular events or cerebritis [4]. 


\section{CASE PRESENTATION}

We present the case of a 65 years-old woman, with medical history of thyroidectomy (for multinodular goiter), severe arterial hypertension, hepatic hemangiomas and L5-S1 disc herniation (for which surgery was performed).

The patient first developed arthralgias, which were attributed to seronegative rheumatoid arthritis, and accused mild cough and dyspnea.

She was later admitted to the hospital for productive cough and moderate exertional dyspnea. Thoracic CT revealed diffuse interstitial pneumopathy. Pulmonary function tests indicated moderate obstruction $(\mathrm{FEV} 1=60 \%)$, while bronchoscopy showed bilateral mucosal edema, hyperemia, and possible bronchiectasis, with bronchoalveolar lavage suggestive of severe alveolar-hemorrhagic syndrome. Testing for mycobacterium tuberculosis was negative. Because the patient started accusing important thoraco-lumbar pain, spinal MRI was performed and revealed compression fractures at the T7 and T12 vertebrae. Results for antibodies against myeloperoxidase (i.e. p-ANCA) were positive and for antibodies against anti-cyclic citrullinated peptides (i.e. anti-CCP) were negative. For this reason, a diagnosis of granulomatosis with polyangiitis was made. Under methylprednisolone $32 \mathrm{mg}$ q.d. and intravenous cyclophosphamide $200 \mathrm{mg}$ q.d., improvement occurred, both clinically and on thoracic CT scan.

During hospitalization, the patient started accusing muscle weakness of the inferior limbs, for which reason she was transferred to our department. Neurologic examination revealed paraparesis 3/5 MRC for the left lower limb and 4/5 MRC for the right lower limb, and diminished pulse of the left dorsalis pedis artery. Brain CT excluded acute ischemic or hemorrhagic lesions. Doppler ultrasound of cervical vessels revealed bilateral carotid atherosclerosis, with a 50-60\% stenosis of the left internal carotid artery, possible occlusion of the right internal carotid artery and a $50 \%$ stenosis of the right carotid bulb and right external carotid artery. Laboratory findings showed hyperglycemia, hypercholesterolemia, and elevated triglyceride levels. Given the rapid progression of paraparesis, electromyographic studies were performed and revealed motor axonal polyneuropathy affecting both upper and lower limbs- the axonal type of
Guillain-Barré syndrome with subacute onset (see Table 1).

TABLE 1. Electromyographic results in our patient

\begin{tabular}{|l|c|c|}
\hline Nerves & CMAP & MCV \\
\hline Tibial (right sided) & Diminished & Normal \\
\hline Common peroneal (bilateral) & Diminished & Normal \\
\hline Median (le & Diminished & Normal \\
\hline Ulnar (bilateral) & Diminished & Normal \\
\hline Nerves & SNAP & SCV \\
\hline & Normal & Normal \\
\hline Surateral) (bilateral) & Normal & Normal \\
\hline Median (bilateral) & Normal & Normal \\
\hline Ulnar (bilateral) & Normal & Normal \\
\hline Muscles & Needle EMG & \\
\hline Right ant & Neurogenic pathway & \\
\hline Le t ? & Normal & \\
\hline Dorsal interosseous & Normal & \\
\hline
\end{tabular}

The patient continued treatment with methylprednisolone $16 \mathrm{mg}$ q.d., oral cyclophosphamide $50 \mathrm{mg}$ q.d and alpha-lipoic acid $600 \mathrm{mg}$ q.d. and was started on intravenous immunoglobulins $15 \mathrm{~g}$ q.d. for 5 days, with improvement of neurological status.

\section{DISCUSSIONS}

As mentioned, both peripheral and central nervous system involvements can be encountered in Wegener's granulomatosis (WG), the first because of vasculitis, and the second due to infiltrating granulomatous lesions [5]. A few cases of spinal cord compression secondary to granulomatous |dural thickening have also been reported [6].

The typical neuropathic manifestations, which are more frequent than the central ones, are mononeuropathy multiplex and multiple cranial neuropathies [7].

The syndrome of mononeuropathy multiplex is asymmetrical and consists of acute or subacute paralysis of multiple individual nerves, simultaneously or serially, due to nerve infarction [8]. Regarding cranial neuropathies, the most affected cranial nerves in WG are the optic, abducens and facial nerves [4].

Sometimes however, a distal symmetrical sensory or sensorimotor neuropathy can be revealed, which can be explained by ischemia-induced axonal degeneration [2].

Peripheral nervous system (PNS) involvement has been estimated by some authors to develop in 
up to $16 \%$ of patients with WG [9]. Others have found a higher prevalence of $23 \%$, nevertheless noting that it occurs less frequently and is less severe than in the other ANCA-associated vasculitides [10].

In one descriptive study of 128 cases of Wegener granulomatosis, patients who developed PNS manifestations were more often males, had a significantly older age at onset of WG (53 versus 44 years-old), a larger disease extent and showed higher titers of ANCA antibodies [11].

Our patient developed an acute motor axonal neuropathy (AMAN), which is the axonal form of Guillain Barré syndrome (GBS).

It has been stated that AMAN is rare in Western countries, but not uncommon in Eastern Asia [12]. A recent study evaluating the geographic variations of GBS, found a higher prevalence in Bangladesh for both the axonal subtype and pure motor clinical form of GBS, as compared to European/ American and Asian countries [13]. AMAN is also known to occur more frequently than the classic demyelinating form of GBS in China, especially during yearly summer outbreaks of GBS [14].

The clinical picture of AMAN is similar to that of classic GBS, however muscle atrophy becomes evident relatively early in the axonal form [8].

\section{REFERENCES}

1. Jennette JC, Falk RJ, Bacon PA, et al. 2012 revised International Chapel Hill Consensus Conference Nomenclature of Vasculitides. Arthritis Rheum. 2013;65(1):1-11.

2. Said G. Vasculitic Neuropaties. In: Said G. Peripheral Neuropathy \& Neuropathic Pain. TFM Publishing Limited. 2015:67-76.

3. Langford CA, Fauci AS. Chapter 326. The Vasculitis Syndromes. In: Longo DL, Fauci AS, Kasper DL, Hauser SL, Jameson JL, Loscalzo J. Harrison's Principles of Internal Medicine. 18th Edition. Volume 2. McGraw-Hill Medical, 2012:2785-2800.

4. Nishino H, Rubino FA, DeRemee RA, et al. Neurological involvement in Wegener's granulomatosis: an analysis of 324 consecutive patients at the Mayo Clinic. Ann Neurol. 1993;33(1):4-9.

5. Holle JU, Gross WL. Neurological involvement in Wegener's granulomatosis. Curr Opin Rheumatol. 2011;23(1):7-11.

6. Roy D, Rao PJ, Phan K, et al. Spinal cord compression from Wegener's granulomatosis: an unusual presentation. J Spine Surg. 2016;2(4):319-323.

7. Asakura K, Muto T. [Neurological Involvement in Wegener's Granulomatosis]. Brain Nerve. 2013;65(11):1311-1317.

8. Allan HR, Martin AS, Joshua PK, Sashank P. Diseases of the Peripheral Nerves In: Allan HR, Martin AS, Joshua PK, Sashank P. Adams and Victor's Principles of Neurology - 11th Edition. McGraw-Hill Educational, 2019:1309-1382.

9. Cojocaru IM, Cojocaru M, Silosi I, et al. Peripheral nervous system manifestations in systemic autoimmune diseases. Maedica (Buchar). 2014;9(3):289-294.
Also, patients with AMAN tend to have more severe symptoms at onset, but the general prognosis does not differ significantly between the two [15].

Development of AMAN has been associated with antibodies against gangliosides GM1, GD1a, GalNac-GD1a and GD1b [16]. Infectious agents, especially Campylobacter jejuni, Cytomegalovirus or Haemophilus influenzae are recognized as potential triggers (through the process of molecular mimicry), by expressing lipooligosaccharides on their surface. These are very similar to the gangliosides found in peripheral nerves and are subsequently targeted by the host's immune system [17].

Regarding therapy, vasculitis neuropathies usually require immunosuppressive treatment of the underlying disorder. Regimens usually begin with corticotherapy. Combined treatment with other immunosuppressive agents, such as cyclophosphamide or rituximab, is useful in refractory or fulminant cases and may also help reduce the dose of corticosteroids $[2,8]$.

\section{CONCLUSION}

In our patient, intravenous immunoglobulins were required, as would have been the treatment of any acute inflammatory polyneuropathy.

\section{Conflict of interest: none declared Financial support: none declared}

10. Cattaneo L, Chierici E, Pavone L, et al. Peripheral neuropathy in Wegener's granulomatosis, Churg-Strauss syndrome and microscopic polyangiitis. J Neurol Neurosurg Psychiatry. 2007;78(10):1119-1123.

11. de Groot K, Schmidt DK, Arlt AC, et al. Standardized neurologic evaluations of 128 patients with Wegener granulomatosis. Arch Neurol. 2001;58(8):1215-1221.

12. Mitsui $Y$, Kusunoki S, Arimura K, et al. A multicentre prospective study of Guillain-Barré syndrome in Japan: a focus on the incidence of subtypes. J Neurol Neurosurg Psychiatry. 2015;86(1):110-114.

13. Doets $A Y$, Verboon $C$, van den Berg $B$, et al. Regional variation of Guillain-Barré syndrome. Brain. 2018;141(10):2866-2877.

14. Ho TW, Mishu B, Li CY, et al. Guillain-Barré syndrome in northern China. Relationship to Campylobacter jejuni infection and anti-glycolipid antibodies. Brain. 1995;118(Pt 3):597-605.

15. Ye Y, Wang K, Deng F, et al. Electrophysiological subtypes and prognosis of Guillain-Barré syndrome in north-eastern China. Muscle Nerve. 2013;47(1):68-71.

16. Ogawara K, Kuwabara S, Mori M, et al. Axonal Guillain-Barré syndrome: relation to anti-ganglioside antibodies and Campylobacter jejuni infection in Japan. Ann Neurol. 2000;48(4):624-631.

17. Kaida K, Ariga T, Yu RK. Antiganglioside antibodies and their pathophysiological effects on Guillain-Barré syndrome and related disorders - a review. Glycobiology. 2009;19(7):676-692. 